\title{
Le garde-manger de Mota Lava (Vanuatu) : un meuble bien particulier
}

The meat safe of Mota Lava (Vanuatu). A very special piece of furniture

La fresquera de Mota Lava (Vanuatu). Un mueble bien peculiar

\section{Virginie Lanouguère-Bruneau}

\section{(2) OpenEdition}

Journals

Édition électronique

URL : https://journals.openedition.org/tc/76

DOI : $10.4000 /$ tc. 76

ISSN : 1952-420X

Éditeur

Éditions de l'EHESS

Édition imprimée

Date de publication : 1 décembre 2003

Pagination : 115-133

ISSN : 0248-6016

Référence électronique

Virginie Lanouguère-Bruneau, « Le garde-manger de Mota Lava (Vanuatu) : un meuble bien particulier », Techniques \& Culture [En ligne], 41 | 2003, mis en ligne le 13 janvier 2005, consulté le 29 septembre 2022. URL : http://journals.openedition.org/tc/76 ; DOI : https://doi.org/10.4000/tc.76

Ce document a été généré automatiquement le 29 septembre 2022.

Tous droits réservés 


\title{
Le garde-manger de Mota Lava (Vanuatu) : un meuble bien particulier
}

\author{
The meat safe of Mota Lava (Vanuatu). A very special piece of furniture \\ La fresquera de Mota Lava (Vanuatu). Un mueble bien peculiar
}

Virginie Lanouguère-Bruneau

1 Aux îles Banks (Vanuatu), l'introduction d'institutions, de pratiques et de valeurs modernes a entraîné un certain nombre de changements -à commencer par l'architecture et l'aménagement de la maison.

2 La maison d'habitation ancienne, décrite par Codrington (1891: 298), est unique. Longue, basse et étroite, elle contient relativement peu d'équipements : un trou et une pile de pierres pour le four, des crochets en bois supportant des sacs d'aliments suspendus hors de portée des rats, des bols en bois ou en coque de noix de coco, des bambous, ni-vinhè (Graminées, Bambusa spp.) pour l'eau, des corbeilles en osier, des outils coincés dans les tuiles. De grands plats en bois avec leur pilon sont entreposés contre les murs. Ils sont employés pour la préparation du ne-let (nalot en bislama), un pudding constitué de taros ou de fruits de l'arbre à pain cuits à l'étouffée, puis écrasés avec le pilon. Le pudding est ensuite découpé avec des couteaux sculptés, réservés aux hommes distingués par la hiérarchie de grades et dans les sociétés secrètes masculines. Ces hommes distingués socialement jouissaient d'une grande autorité en raison de leur mana, une force originaire des êtres du cosmos avec lesquels ils étaient liés. La maison contient également, près de l'une des portes, au-dessus d'un foyer creusé dans le sol, un garde-manger.

3 De nos jours, les villageois possèdent souvent deux maisons: une cuisine (avec les ustensiles modernes, cocottes, couteaux, verres, assiettes, couverts, etc.) ; un logement pour dormir et entreposer les affaires personnelles (vêtement, couvertures, éventuellement papier, crayons, etc.). Ce logement contient parfois une table, des bancs, ainsi que des objets modernes (lampes, coffres, valises, etc.). Le garde-manger 
tend à disparaître dans les maisons contemporaines, et n'est plus visible aujourd'hui que dans les maisons des personnes âgées, qui vivent encore dans une maison unique.

Plusieurs études ont été menées aux îles Banks² mais le garde-manger, no-motow dans la langue de Mota Lava, n'a jamais fait l'objet de travaux spécifiques. Lors d'une enquête de terrain réalisée en 1997-199833, il m'est apparu que ce meuble avait une place très particulière. Il existe encore quelques garde-manger à Mota Lava, mais ils ne sont plus fabriqués aujourd'hui. Leur fabrication requiert une technique précise, connue seulement de certains hommes dont le nombre diminue à mesure que les années passent. Dans les temps anciens, ces hommes recevaient de la monnaie de coquillage, en moyenne deux «troncs » (yovtogotgo vôyô), soit environ 40 Vatus, selon la taille du garde-manger ${ }^{4}$. Aujourd'hui, l'absence de transmission de la technique de fabrication de cet objet valorisé, le manque de monnaie (tant ancienne en coquillage qu'actuelle en Vatus) et aussi l'envoi depuis les villes de coffres ou autres valises modernes, rendent inutile et désuète la réalisation de nouveaux garde-manger. Le savoir-faire en la matière risque donc d'être perdu pour les générations futures. Pourtant, il reste d'une grande valeur sociale, quant à sa fabrication, ses matériaux et son utilisation.

Marcel Mauss (1947: 76) conseillait, pour étudier la maison d'habitation, d'en faire établir un modèle réduit afin de comprendre les rapports entre les différentes parties qui la composent. Konsis Alféa, un homme de plus de 70 ans connaissant la technique de fabrication du garde-manger, en a réalisé une miniature ${ }^{5}$ (photo 1 ), qui m'a permis d'observer la chaîne opératoire de sa construction.

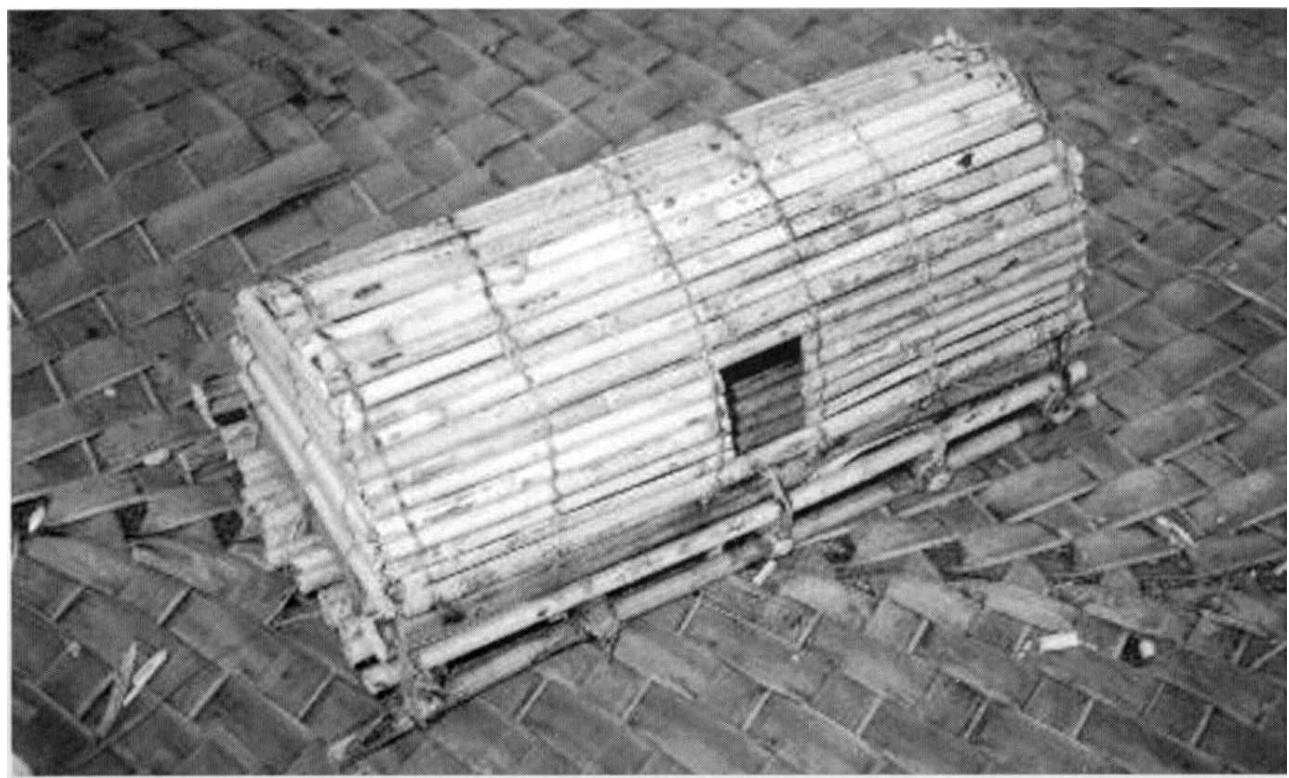

Photo 1. Garde-manger miniature réalisé par Konsis Alféa en 1998

6 L'analyse technologique d'un objet passe en effet par la description de la chaîne opératoire relative à sa fabrication, dont Robert Cresswell (1976: 13) donne une définition simple et pertinente : «Une chaîne opératoire est une série d'opérations qui transforme une matière première en un produit, que celui-ci soit objet de consommation ou outil ». Je propose ainsi de suivre celle de la fabrication du gardemanger.

7 Yves Delaporte (1986) note qu'un ethnologue qui décrit une chaîne opératoire a toutes les chances d'être confronté à des variantes. Je n'ai pu assister qu'à la fabrication d'un 
garde-manger en miniature, aussi les éventuelles variantes resteront-elles indéfinies pour cette première approche. Étant donné l'importance sociale de cet objet, il est fort possible que certains gestes qui avaient autrefois un sens aient été omis par Konsis Alféa parce qu'il n'en avait pas connaissance, ou parce qu'ils étaient inutiles pour la réalisation d'une miniature, ou encore parce que le lieu était inadéquat ou que les conditions de réalisation n'étaient pas réunies.

On peut supposer que la fabrication d'un garde-manger revêtait autrefois une valeur similaire à celle des masques des sociétés secrètes, qui transformaient les hommes du commun en hommes de distinction. Les îles Banks sont reconnues pour être le lieu d'origine des masques na-tmat ${ }^{6}$ fabriqués secrètement par les hommes en ayant acquis le droit, et ce savoir accroît leur force $\mathrm{mana}^{7}$. Ces derniers se rendent en forêt en un lieu tenu secret pour "faire sortir les ancêtres"; les masques sont censés donner une apparence visible à des ancêtres invisibles. Il est possible enfin que la fabrication du garde-manger ait exigé le recours à des pratiques magiques, aujourd'hui oubliées. La transmission d'une technique de fabrication est parfois utile pour éclairer ou approfondir la compréhension de certains gestes (Delaporte 1986), mais je n'ai pu l'effectuer. Quoi qu'il en soit, le présent travail reste important dans la mesure où de moins en moins d'hommes sont aujourd'hui capables de le fabriquer'.

\section{Description et fabrication}

Le garde-manger est décrit en des termes qui le rapprochent d'un être vivant. Il a un visage, na-ngon, qui correspond au côté où se trouve la porte et un dos, ne-klan, le côté opposé. Les deux faces latérales sont considérées comme des postérieurs, nu-qutiyg (schéma 1). Il est comme « une maison dans la maison », un abri dans l'abri, et duplique ainsi le rôle protecteur de l'habitation. Sa technique de fabrication confirme sa valeur, tant en raison de certains gestes, des matériaux utilisés, des signes distinctifs qu'il porte, que de son usage comme lieu de protection de biens valorisés. Je commencerai donc par décrire la chaîne opératoire de sa fabrication, en indiquant les gestes et les matériaux chargés de sens, qui seront analysés dans un deuxième temps.

Comme le note Hélène Balfet (1986), une chaîne opératoire peut être découpée en différentes unités, le premier niveau de découpage étant la phase. On peut ainsi " découper » la fabrication d'un garde-manger à Mota Lava en six grandes phases, ellesmêmes décomposées en séquences puis en opérations.

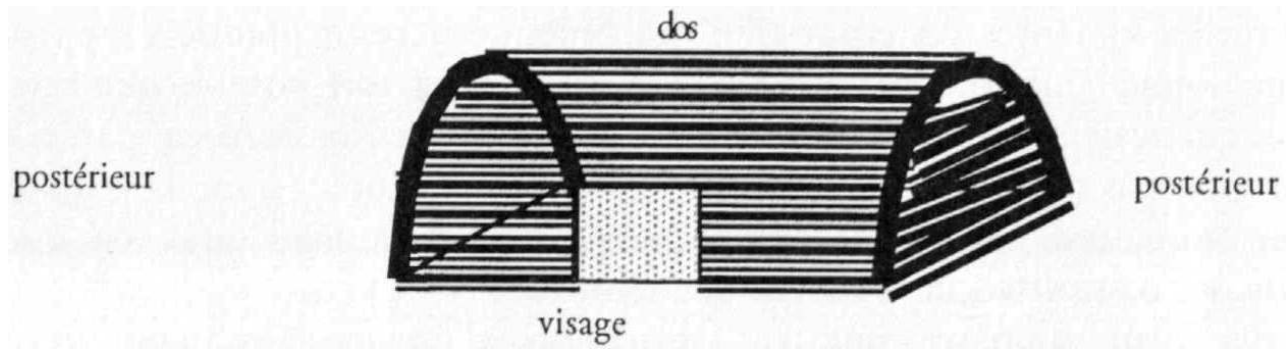

Schéma 1. Le garde-manger, un être vivant 


\section{Phase 1. La fabrication des liens (cordes et cordelettes)}

11 La première phase consiste à fabriquer des cordes et cordelettes qui serviront à assembler les différentes parties du garde-manger. Leur matière première est le rotin, na-gawol (Flagellaria spp. ${ }^{9}$ ), ou bien l'écorce d'hibiscus, na-gavey (Malvacées, Hibiscus tiliaceus $)^{10}$. Ces liens sont également utilisés pour la construction des maisons et la fabrication des masques na-tmat dans les sociétés secrètes (photo 2).

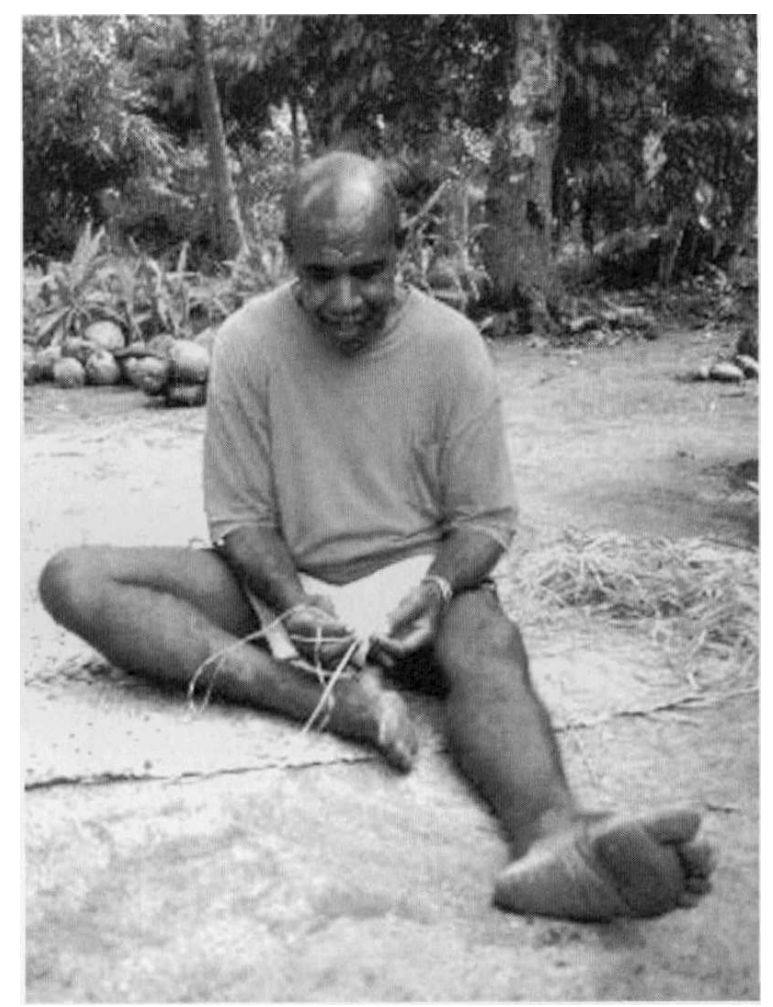

Photo 2. Fabrication des cordes et cordelettes en Hibiscus

Pour les cordes en hibiscus, l'écorce de l'arbre est aujourd'hui prélevée avec un couteau; autrefois on employait des herminettes, des coquillage ou on se passait d'outils. Les fibres qui se trouvent à l'intérieur de l'écorce sont détachées à la main et mises à sécher au soleil. En séchant, elles se séparent en filaments, qui sont ensuite torsadés ensemble, généralement sur la plante du pied (photo 3). 


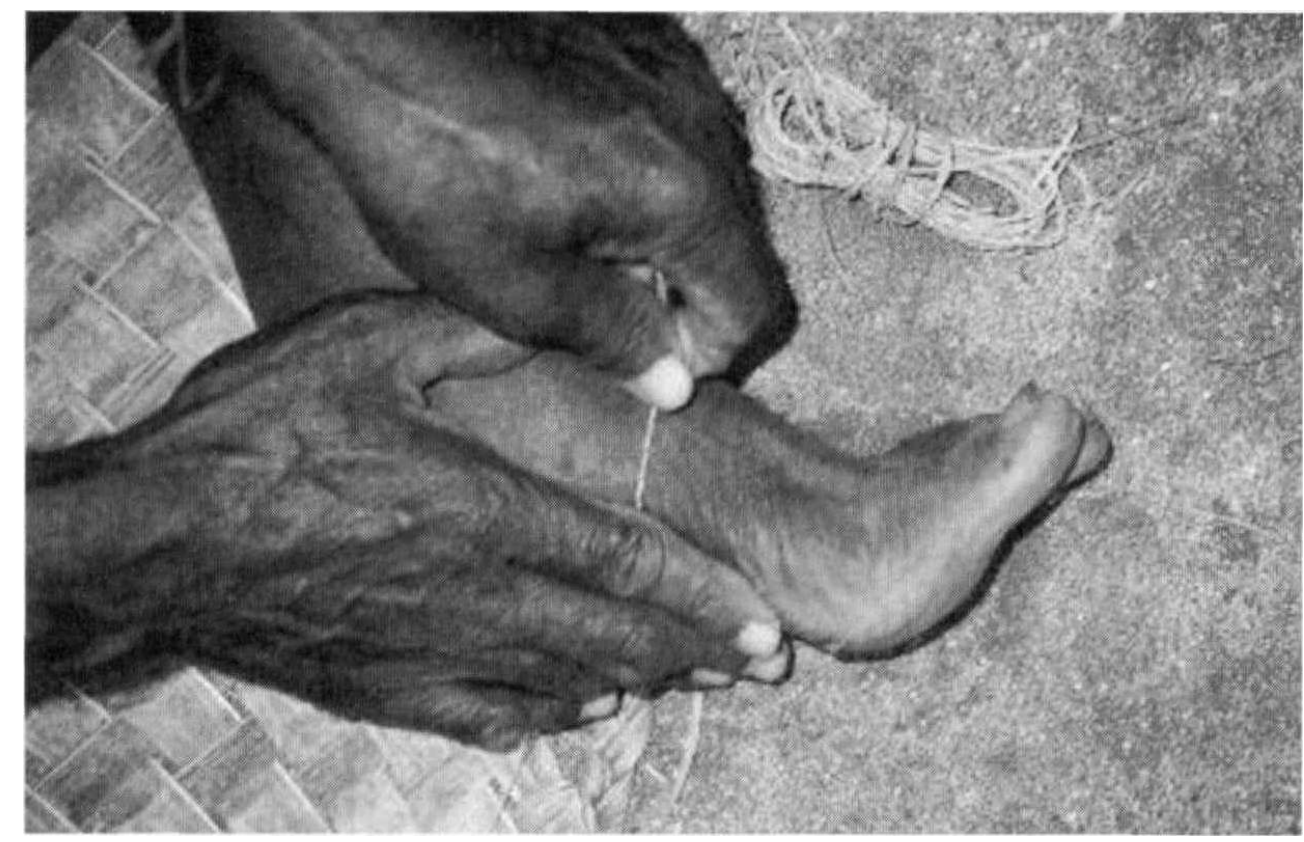

Photo 3. Les cordelettes en fibre d'hibiscus sont roulées sur le pied

13 L'expression employée pour désigner cette technique est gal ôh. Elle se compose de deux termes : gal qui signifie nouer, attacher fortement, et le verbe ôh qui décrit l'action de rouler ensemble plusieurs filaments. L'action de rouler les fibres sur la plante $d u$ pied se dit gal labalbe. Le pied est d'usage courant dans l'artisanat, par exemple pour la confection de paniers, d'éventails et autres nattes par les femmes. Lorsqu'il roule des cordes et cordelettes, l'artisan crache souvent dans ses mains pour humidifier les fibres. Il s'agit là d'un geste chargé de sens, on l'effectue dans les sociétés secrètes et pour toute action qui donnera aux hommes un surcroît de mana ou de prestige face aux membres de la communauté. Plusieurs longueurs de cordes et cordelettes sont nécessaires : les plus petites mesurent de 20 à $30 \mathrm{~cm}$, les plus grandes peuvent atteindre $2,50 \mathrm{~m}$.

\section{Phase 2. La fabrication du socle}

14 Une fois les cordes et cordelettes prêtes, l'artisan peut passer à la deuxième phase, qui consiste à fabriquer le socle du garde-manger. La fabrication du rectangle de base constitue la première séquence de la deuxième phase. L'artisan utilise les branches de deux arbres littoraux nommés nê-'nêy (Simarubacées Suriana maritima ${ }^{11}$ ) et na-'mah (Sapindacées Allophyllus timorensis, cf. François à paraître) -qui est également le nom d'un poisson. Je n'ai pas connaissance d'autres arbres employés pour cette partie du socle. Lors de la deuxième séquence, l'artisan pose des traverses confectionnées à partir du même bois. La base rectangle et les traverses sont attachées avec les petites cordelettes, ce qui forme une « échelle de bois » (photo 4). 


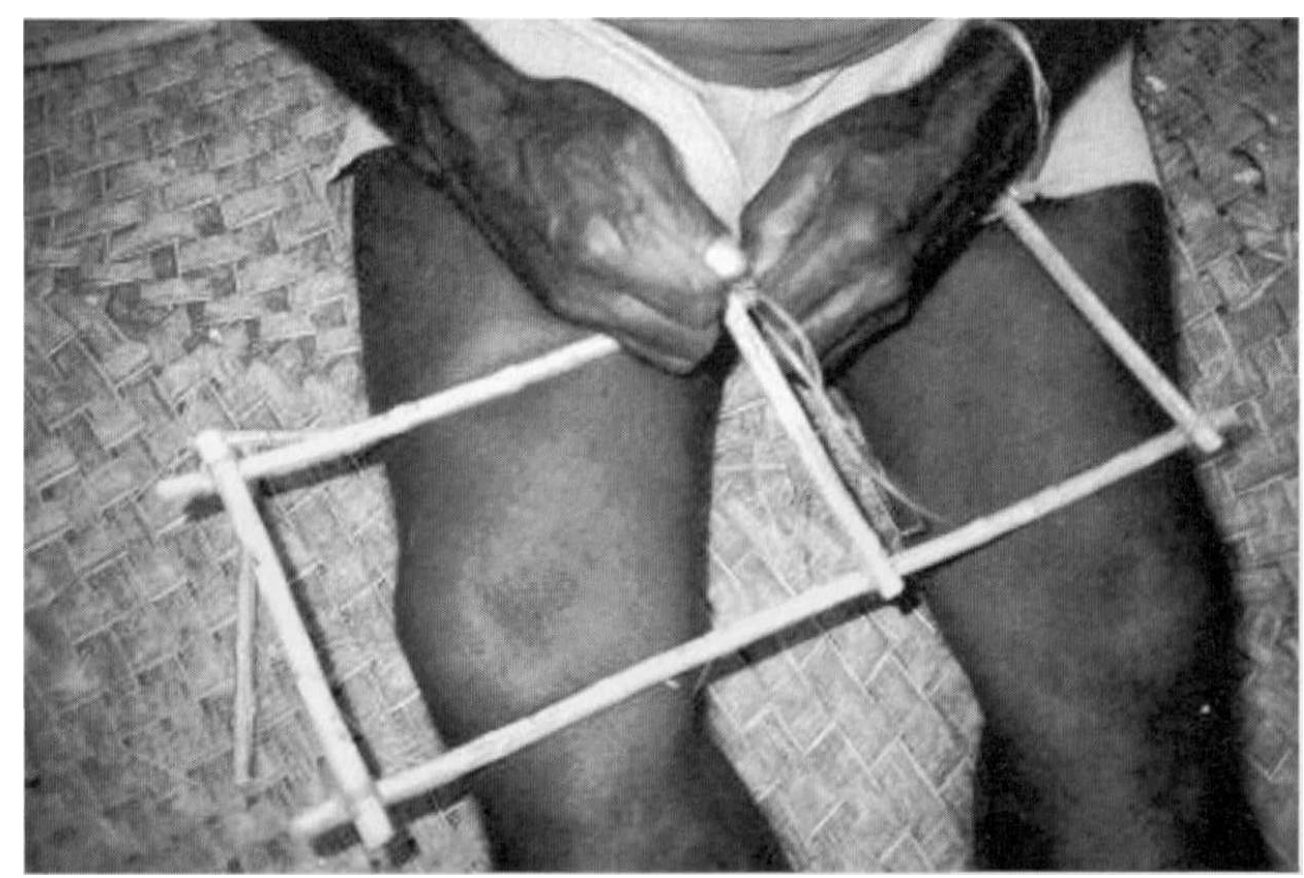

Photo 4. L'échelle de base du garde-manger et les traverses

15 Les nœuds sont ensuite enduits de la sève de l'arbre à pain na-mte (Moracées Artocarpus altilis), qui durcit les fixations. Il existe des arbres à pain plus efficaces que d'autres à cet effet (nebew taban, na-mte mêsmês ${ }^{12}$ ), car leur sève est plus collante. La connaissance des arbres à pain les plus appropriés était jadis enseignée aux hommes lors de la fabrication des masques na-tmat. Aucune autre technique ne semble exister pour consolider les nœuds et les fixer durablement. De plus, ce procédé ne semble pas être employé ailleurs et il est probable qu'il faisait partie d'un savoir réservé aux hommes initiés dans les sociétés secrètes.

La séquence suivante (la troisième) consiste à recouvrir l'échelle de base de morceaux de roseaux, no-tog (Graminée Miscanthus floridulus), disposés dans le sens de la longueur (photo 5). Aucun autre végétal n'a été signalé par Konsis Alféa. Nous verrons que le roseau a une valeur sociale particulière à Mota Lava. 


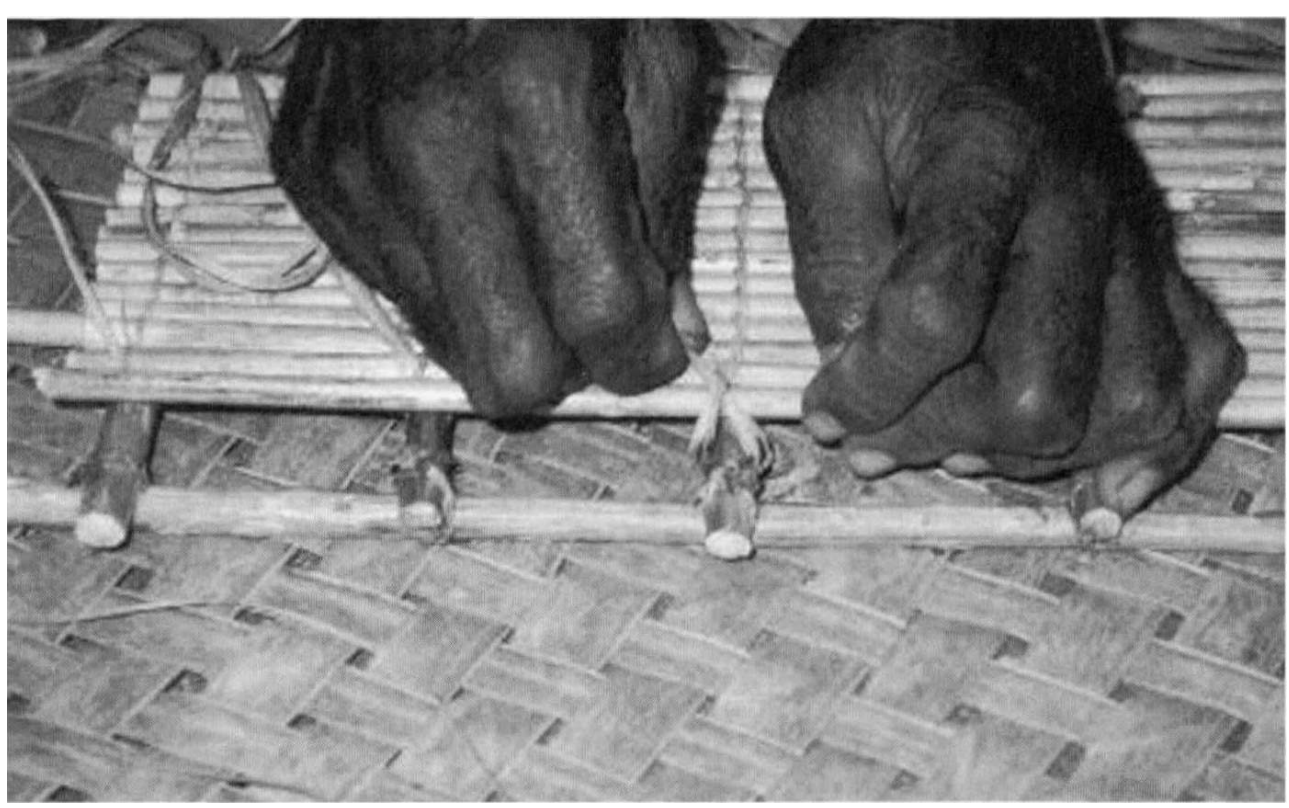

Photo 5. Le socle du garde-manger

17 Il existe un nom pour chaque partie du socle: le rectangle de base et les traverses forment, littéralement, le lit du garde-manger, ne-qe no-motow; chaque traverse possède aussi un nom, mais Konsis Alfea n'a pu s'en souvenir.

18 Il existe une étape supplémentaire qui consiste à rajouter une rangée de rondins de bois ${ }^{13}$ en bordure du socle, sur lesquels seront enfoncées des branches en arc de cercle. Cela correspond à l'autre partie du garde-manger, l'armature. Ce pourtour en bois tendre est visible sur la photo 7 et sur la photo 8.

19 Le socle du garde-manger compte ainsi quatre niveaux avec la base rectangle, les traverses de l'échelle, les lattes longitudinales et le pourtour en bois tendre.

\section{Phase 3. L'armature du garde-manger}

20 La troisième phase de la chaîne opératoire consiste à monter l'armature du gardemanger. Konsis Alféa a confectionné les arceaux du garde-manger miniature, uniquement avec du roseau. Deux autres types de bois sont employés pour un gardemanger de taille normale.

L'artisan doit disposer de branches de sagoutier, no-yot (Palmiers Metroxylon warbuggii Becc.), ou de branches de l'arbre na-'mah, qu'il trouve en forêt ou en bord de mer. Il enlève l'écorce des branches pour éviter qu'elle ne soit mangée par les insectes ${ }^{14}$. branches de ces deux arbres sont chauffées sur le feu, yat bah, afin de les assouplir et les courber (wolow) en arc de cercle. Le pourtour du socle, constitué du rectangle de rondins de bois, est percé de trous sur l'avant (visage) et l'arrière (dos) du gardemanger, pour y fixer les arceaux (photo 6). Ces derniers sont en outre attachés à l'aide des différentes cordes et cordelettes, puis on durcit les nœuds avec la sève de l'arbre à pain. Au sommet de cette armature est fixée une "fausse " poutre faitière, nommée neqethon gal dans la langue de Mota Lava, terme construit à partir de l'article ne-, de qet (« tête »), de hon (« dernier ») et de gal (« mentir »). 


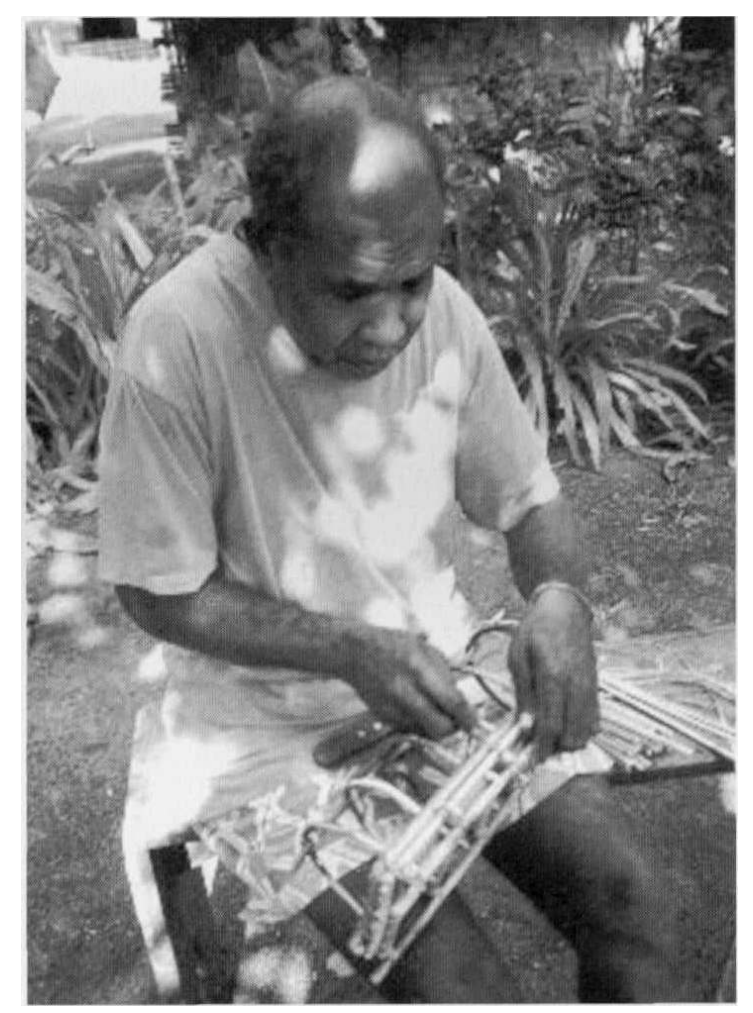

Photo 6. Les arceaux de l'armature

\section{Phase 4. Les murs extérieurs (postérieurs) du garde-manger}

La phase suivante consiste à monter les murs du garde-manger à partir de bambous collectés en forêt, coupés dans le sens de la longueur puis tressés ${ }^{15}$. Ce même procédé est employé dans la construction des maisons d'habitation. Il existe différents tressages représentant divers motifs (quadrillage, losanges, etc.) appropriés au rang de l'homme à qui le garde-manger est destiné. S'il s'agit d'un « haut gradé », le tressage est nommé la'm; il requérait autrefois un savoir-faire particulier. Pour le garde-manger miniature, Konsis Alféa a choisi des roseaux pour monter les murs (photo 7), car les bambous auraient été trop larges. Pour un garde-manger de taille normale, le bambou reste le matériau idéal. Les palmes de cocotier na-mtig (Palmiers Cocos nucifera) pourrait être utilisées, comme elles le sont pour les murs des maisons de brousse. Cependant, il s'agit là de maisons provisoires et cette technique ne pourrait s'appliquer au garde-manger. 


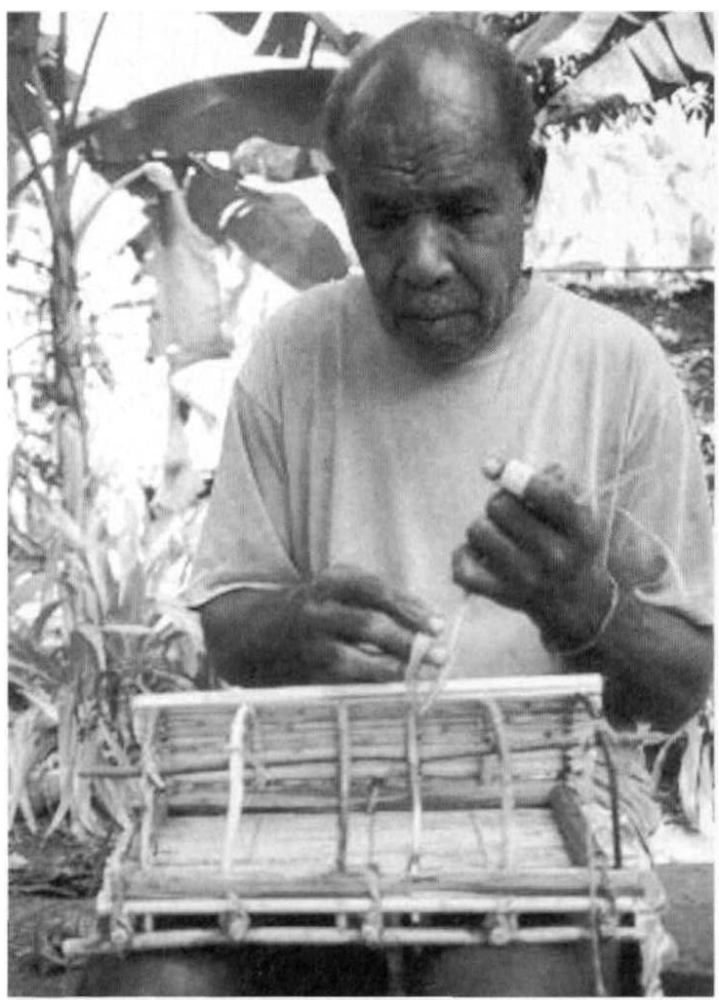

Photo 7. Le montage des murs du garde-manger

Sur la façade, le "visage ", na-ngon, l'artisan laisse une ouverture où sera fixée une porte $^{16}$, Pour les côtés, les "postérieurs ", nu-qutiy, la fermeture s'effectue en deux temps. L'artisan fixe d'abord des poteaux, généralement taillés dans des branches de l'arbre $n$-a'mah et plantés dans le rectangle de rondins de bois tendre, comme pour les arceaux. Il est possible que d'autres arbres soient choisis mais aucun relevé systématique n'a été établi lors de cette première observation. L'artisan monte ensuite les murs en bambou comme pour le reste du garde-manger (photo 8).

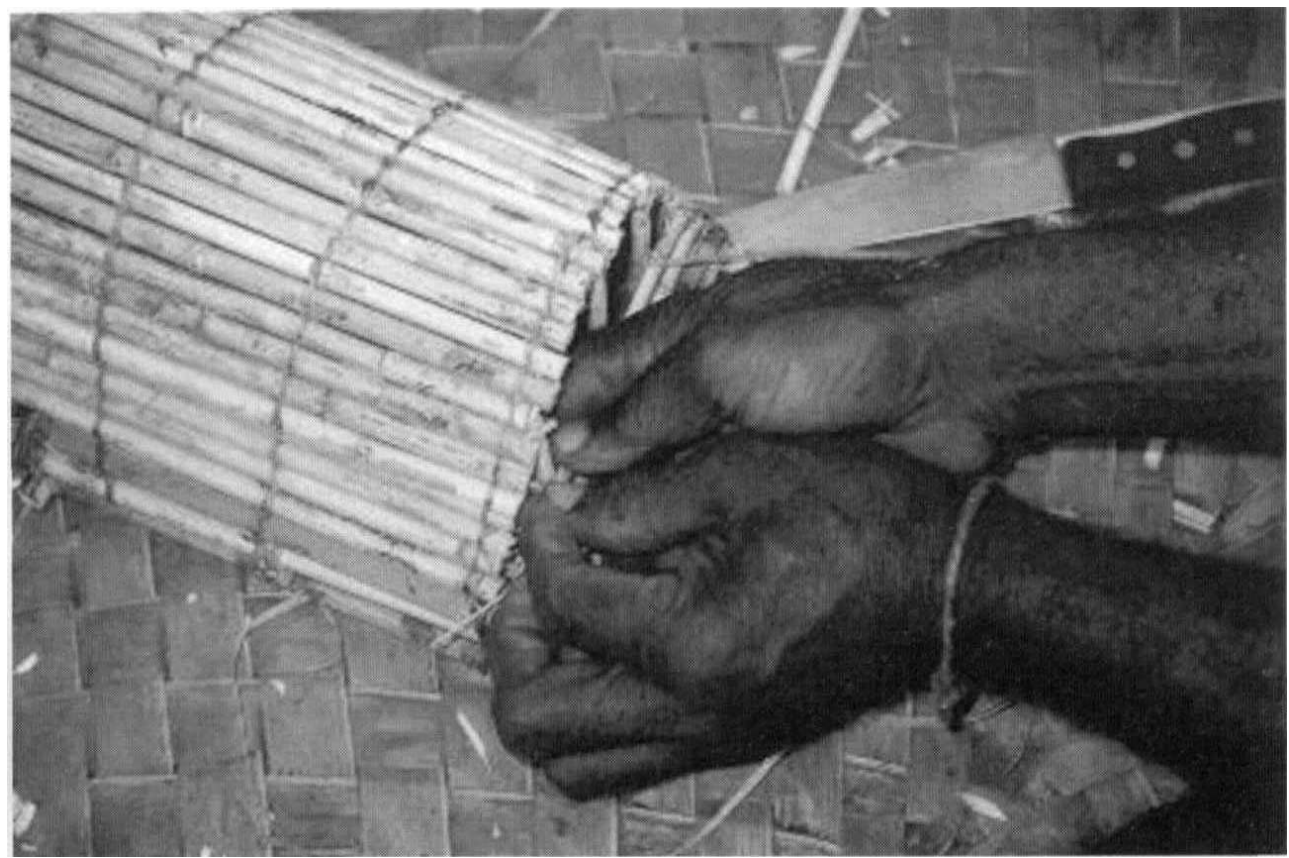

Photo 8. Fermeture des murs sur les côtés 


\section{Phase 5. Le tapissage intérieur} est tapissé de feuilles na-gavag, une variété de pandanus, no-yopdege (Pandanacées, Pandanus spp.), dont l'épine dorsale est ôtée, ou encore avec des feuilles de sagoutier utilisées également pour les tuiles des maisons. Le feuilles sont préalablement séchées au soleil ou éventuellement dans un lieu couvert, avant d'être posées. Aucun autre végétal ne semble pouvoir être employé pour ce tapissage intérieur, et nous reviendrons dans la seconde partie sur l'importance de ces deux végétaux. Plusieurs termes décrivent l'action de tapisser, lil goy, vatqep, mais celui employé pour « couvrir avec des feuilles sèches» est yoq goy. Les "postérieurs» du garde-manger sont également tapissés.

\section{Phase 6. La mise en place du garde-manger}

Le garde-manger est ensuite installé dans la cuisine. On le pose sur des poteaux fichés dans le sol, dont l'extrémité est fourchue (photo 9). Ces poteaux doivent être en bois dur (sans autre précision), pour qu'ils résistent au feu. Le garde-manger est en effet situé au-dessus du petit foyer, d'usage quotidien.

26

À l'intérieur, sont entreposés des biens valorisés. Autrefois, la monnaie de coquillage, les racines de kava na-ga (Pipéracées, Piper methysticum) réservées aux hauts gradés, les velles ne-vel (Lécythidacées, Barringtonia edulis) et nangailles ne-'ney (Burseracées Canarium indicum) séchées, gardées pour les cérémonies et les fruits de l'arbre à pain séchés, ne-key, étaient conservés dans ce meuble, de même que les os de certains défunts. En outre, un garde-manger construit spécialement à cet effet servait à entreposer les momies des très hauts gradés dans la forêt, afin d'éviter la profanation de leur tombe. Parfois, seuls leurs os, véhicules de force mana, y étaient déposés.

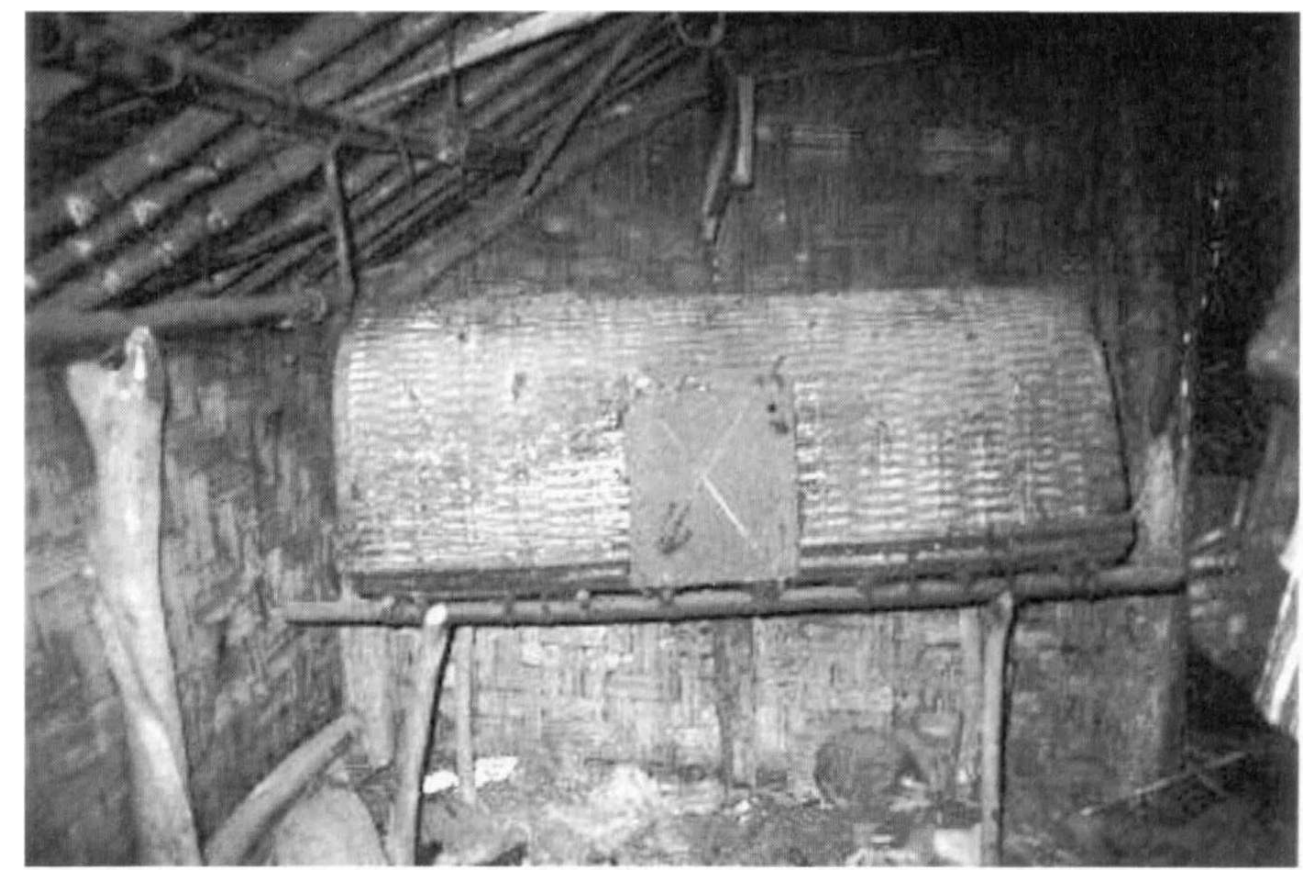

Photo 9. Le garde-manger no-motow

Techniques \& Culture, 41 | 2003 

de Konsis Alféa. Nous ne disposons pas d'informations quant aux lieux de fabrication. Cependant -et d'autant plus si la fabrication du garde-manger requérait dans les temps anciens des pratiques magiques-, il est tout à fait possible que certaines phases aient été réalisées au sein de la maison masculine située dans le village ou bien dans celle en forêt, encore plus fermée. Pour le garde-manger miniature, Konsis Alféa a assuré à lui seul toute la fabrication, y compris l'approvisionnement en matériaux -en forêt ou en bord de mer.

Aussi limitée que soit la description technique présentée dans cet article, on peut constater que la fabrication du garde-manger relève d'un savoir-faire spécifique. Il est l'apanage de quelques hommes initiés, et ses décorations varient selon le rang social de la personne à qui il est destiné.

\section{Un meuble très valorisé...}

\section{Par les gestes et les techniques...}

Un geste souvent employé par Konsis Alféa, tout au long de la fabrication du gardemanger, consiste à se cracher dans les mains, par exemple pour humidifier les cordes et les rouler. Selon lui, ce geste est également fréquent dans la maison de brousse des hommes, le na-halgoy, lorsque les initiés font sortir les masques. Jadis, cette institution du na-halgoy, terme souvent rendu dans la littérature par "sociétés secrètes ", était associée à une autre institution, la hiérarchie des grades, nô-sôq, aujourd'hui disparue. Les décorations acquises dans le na-halgoy et les attributs des rangs obtenus dans la hiérarchie nô-sôq étaient exhibés lors de fêtes publiques (no-kolkol). Ces trois sources de distinction formaient les institutions statutaires. Les hommes qui y progressaient, acquéraient des liens avec les êtres du cosmos et, par là, de la force mana. Ainsi participaient-ils rituellement à la reconduction de la société. Ils aidaient notamment les ancêtres à parcourir le chemin entre le monde des vivants et celui des morts. Le fait d'enduire les nœuds de sève d'arbre à pain est aussi une technique pratiquée dans les sociétés secrètes, et l'emploi de cette sève semble être propre à la fabrication d'objets valorisés. Notons encore le recours au lait de coco ou à la chair râpée pour nettoyer les mains, un geste également effectué lors des réunions des sociétés secrètes.

Le fait que l'on retrouve des techniques communes à la fabrication des masques na-tmat du na-halgoy et du garde manger montre qu'il s'agit d'un même type de réalisation, la fabrication d'objets valorisés, en relation avec les êtres du cosmos, qui procurent $d u$ mana aux hommes socialement distingués.

\section{...Et par ses matériaux}

31 De nombreuses lianes sont employées à la fois pour la fabrication du garde-manger, et pour les représentations na-tmat du na-halgoy, qui donnent une apparence visible à des ancêtres invisibles pour le commun des mortels. Des végétaux comme l'hibiscus, le roseau, le sagoutier, le bambou, le pandanus, principalement, et dans une moindre mesure, le cocotier et l'arbre à pain, ont des emplois divers et véhiculent une valeur sociale particulière.

Techniques \& Culture, 41 | 2003 
32 La fleur de l'hibiscus, dont l'écorce donne des cordelettes, était un attribut des hommes qui avaient acquis des distinctions sociales dans les institutions statutaires (na-sâq, nahalgoy, na-kolkol). La fleur comme les fibres de cet arbuste témoignent d'une part d'un lien social entre les hommes qui appartiennent aux mêmes institutions, et d'autre part d'un lien physique - puisque les fibres servent à attacher les différentes parties du garde-manger.

33 Le roseau, dont est composé le socle du garde-manger, entre dans la construction de la maison d'habitation. Il sert notamment à fabriquer les murs d'une chambre intérieure et à confectionner les tuiles du toit (avec le sagoutier). Le roseau était un support de la monnaie de coquillage qui circulait entre les hommes socialement distingués, pour transmettre un message de paix. Ces coquillages venaient de la mer, où vivent certains esprits et ancêtres, et la monnaie renfermait de ce fait la force mana que procurent ces derniers. Le roseau peut être aussi le support de feuilles valorisées, comme les crotons na-sas (Euphorbiacées, Codiaeum variegatum). Leur présence pose un interdit en un lieu particulier. On l'emploie également comme tuteur pour les ignames dont le mythe raconte qu'elles sont issues du corps d'une grand-mère. Il est dit, enfin, que certaines personnes qui se déplacent la nuit vont danser autour d'un arbre en enfourchant un roseau (comme les sorcières de chez nous enfourchent leur balai). Le roseau assure donc une relation entre le monde des vivants et celui des êtres du cosmos (esprits et ancêtres). Il est un support pour les messages, les biens ou les personnes qui détiennent de la force mana.

34 Avec des branches de sagoutier, on peut confectionner les arceaux du garde-manger et les murs des côtés (les "postérieurs »), tandis qu'avec ses feuilles on en tapisse l'intérieur. Dans d'autres constructions (maisons d'habitations, maison des hommes, cuisine des femmes, etc.), les feuilles sont employées (avec le roseau) pour la confection des tuiles végétales et sur la poutre faîtière, ainsi que pour celle de la porte d'entrée de la maison d'habitation ancienne. Enfin, elles décorent la maison publique des hommes lors d'une cérémonie nommée wuwuhyaw, célébrée à l'occasion de l'acquisition du septième grade de la hiérarchie nô-sôq. Leur présence annonce publiquement que la maison est fermée en raison de la prise de grade qui s'y déroule. Le sagoutier apparaît ainsi comme un matériau qui protège et isole de l'extérieur. Là encore, l'emploi possible de ce végétal confirme le rôle essentiel du garde-manger.

Le bambou est employé pour la construction des murs extérieurs du garde-manger, et pour les murs des maisons. Les différents motifs de tressage attestent de la distinction de statut entre les gens du commun et les gradés. Le bambou intervient de plus dans les jeux funéraires: plusieurs hommes tiennent un grand bambou que les ancêtres investissent et font bouger. Certains hommes peuvent même «décoller ». Il est également employé pour transporter l'eau dans laquelle vivent certains esprits et ancêtres ${ }^{17}$. De la sorte, le bambou devient un récipiendaire des ancêtres. Comme pour les maisons, le bambou des murs extérieurs du garde-manger crée une barrière entre intérieur et extérieur. Les biens entreposés dans le garde-manger relèvent d'une relation particulière avec les êtres du cosmos, qu'ils soient des êtres vivants, ou bien qu'ils aient la forme d'ancêtres ou d'esprits.

Le pandanus est aussi un arbre de grande valeur à Mota Lava. D'une part, les événements du mythe d'origine des deux moitiés matrilinéaires exogames se déroulent sur un pandanus dont une jeune fille doit cueillir les fruits. D'autre part, ses feuilles servent à tresser des nattes utilisées comme couche et comme linceul. Enfin, les 
hommes des rangs élevés de la hiérarchie nô-sôq acquièrent le droit de porter une robe nommée na-'malhey, qui compte quatre pans en feuilles de pandanus. Ce droit implique un rang dont l'importance sociale s'étend au niveau régional (et non plus seulement local). L'emploi du pandanus témoigne chaque fois d'un changement: de système de mariage avec l'introduction des deux moitiés, d'état lors du sommeil ou après la mort, de rang et de régionalisation des relations pour les hauts gradés. Le pandanus est lié à la transformation d'une personne dans ses relations avec une entité plus grande: l'ensemble de la parenté dans le cas des moitiés matrilinéaires, le cosmos dans le cas du sommeil et du décès, le monde des vivants avec l'extension à l'échelle régionale. Comme le sagoutier, il tapisse l'intérieur du garde-manger, et en fait une antre qui abrite des bien relevant de relations sociales et cosmiques valorisées.

L'utilisation de la sève de l'arbre à pain rappelle l'importance des fruits de cet arbre pour les échanges inter-îles. En effet, dans les temps anciens, les îles Banks étaient spécialisées dans la production de différents biens. Mota Lava était réputée (et l'est toujours même si ces échanges n'ont plus lieu) pour la quantité et la qualité de ses arbres à pain. Ses habitants détiennent toujours le savoir-faire de la transformation des fruits de l'arbre à pain en biscuits nommés ne-key, qui se conservent plusieurs années. Notons enfin que le cocotier est, lui aussi, un arbre de très grande valeur aux îles Banks. Il intervient dans une moindre mesure dans la fabrication du garde-manger. Cet arbre est présent dans tous les grands moments de la vie sociale : un accouchement difficile, un mariage (dans la compensation matrimoniale), les préparations culinaires valorisées, lors des funérailles (il est interdit de le consommer pour les proches du défunt), etc. Par ailleurs, le cordon ombilical d'un enfant est enterré au pied d'un cocotier, près du monde des morts (amno). Le mythe d'origine de la noix de coco raconte qu'elle vient de la germination d'une femme-serpent tuée par son gendre. L'eau de coco est le sang de cette femme-serpent. Nettoyer la sève de l'arbre à pain avec la chair ou le lait de la noix de coco revient ainsi à se laver les mains avec le sang d'un ancêtre féminin d'importance évidente dans une société matrilinéaire, pour la succession des générations.

\section{Le garde-manger : un lieu de protection}

39 Jadis, seuls les hauts gradés buvaient du kava. Ses racines étaient enveloppées dans des feuilles ombrelles, ne-yendip (Palmae, Licuala grandis Wendl), et mises à sécher au-dessus du feu ou dans le garde-manger pendant environ un an. De jeunes hommes mâchaient ensuite le kava séché (naga meye'n), et le jus que l'on en extrayait était déversé dans une coupe de noix de coco. Le haut gradé buvait une à deux coupes de ce kava non dilué et de ce fait très fort. Grâce à cette boisson rituelle, les hauts gradés entraient en relation avec les ancêtres et les esprits. Le kava était donc une plante de grande valeur, conservée dans ce lieu hautement valorisé que constituait le garde-manger.

Dans celui-ci, étaient également entreposées les nangailles et les velles séchées, utilisées pour préparer des plats partagés lors de diverses cérémonies (naissance, mariage, funérailles, etc.) ou entre les « hauts gradés ». Deux des îles Banks, Mere Lava et Merig, sont réputées pour leur grand nombre de velles, dont une certaine quantité était issue des échanges inter-îles, peut-être contre des fruits de l'arbre à pain. Les relations entre les îles étaient très utiles pour obtenir des biens indispensables à la promotion sociale des hommes ainsi qu'aux cérémonies. Plus encore, disposer de biens 
acquis dans d'autres îles prouvait qu'un village avait de "hauts gradés » capables de nouer des relations au niveau régional -disposant donc d'une grande force mana. La valeur sociales de ces nangailles et de ces velles obtenues par les échanges inter-îles justifiaient qu'elles fussent conservées dans le garde-manger.

De même, les habitants du banc de sable Rowa produisaient une monnaie de coquillage de grande qualité, qui circulait dans toutes les îles Banks, et au-delà. Les femmes de Mota Lava savaient également fabriquer la monnaie de coquillage, nécessaire à l'acquisition de distinctions sociales et à la promotion dans les institutions statutaires. La monnaie dont un homme disposait, quelle que fût son origine, était entreposée dans le garde-manger. Le contact indirect avec la fumée du foyer du dessous la noircissait, ce qui lui donnait un surcroît de valeur. Une monnaie blanche et neuve avait en effet une moins grande valeur qu'une monnaie noircie, ayant un certain passé, une histoire. Là encore, le garde-manger protège un bien valorisé, plus : lui insuffle de la valeur.

Selon les informateurs, la décoration ne-wetbôm propre au septième grade na-tvôsôqlap, faite avec des colorants végétaux puis mise à sécher au soleil, comme l'écorce et les lianes utilisées pour fabriquer les cordelettes, était également conservée dans le gardemanger. On emballait cette décoration dans une feuille ombrelle ne-yendip pour la protéger de la fumée (à l'inverse de la monnaie) et en conserver la couleur. Cette décoration de la hiérarchie de grades trouve, comme de nombreux biens valorisés, un lieu de protection dans le garde-manger. Ce dernier la protège et la préserve de la détérioration naturelle et du regard des gens du commun.

Codrington (1891 : 267) relève, enfin, qu'aux îles Banks, les défunts ordinaires étaient enterrés dans la forêt, non loin du village, tandis que les très hauts gradés pouvaient être inhumés dans le village, près de la maison publique des hommes, afin d'éviter la profanation de leur tombe pour récupérer leurs os chargés de mana. La tombe était ouverte après 50 ou 100 ans, et les ossements étaient cachés dans la forêt ou suspendus dans la maison. Aujourd'hui, les gens de Mota Lava se rappellent que les os de ces hauts gradés étaient également conservés dans le garde-manger au-dessus du foyer. De plus, comme je l'ai déjà signalé, Codrington indique que certains corps n'étaient pas enterrés, mais laissés dans la forêt dans un garde-manger fabriqué pour l'occasion.

Le garde-manger, abri dans l'abri, n'est pas un lieu anodin, car il se trouve-en étroite liaison avec l'ensemble des êtres du cosmos. En témoignent le vocabulaire qui le décrit, lequel évoque le corps humain, et les végétaux importants qui entrent dans sa fabrication. Le garde-manger a sa place dans la maison; à l'abri de l'extérieur, il protège les biens valorisés de la famille. Il est installé dans la cuisine, et plus particulièrement au-dessus du petit foyer, où il subit les émanations du feu. Celui-ci transformant les nourritures participe de ce fait à la transformation des êtres du cosmos. La suie issue du feu accroît la valeur de certains biens déposés dans le gardemanger. D'autres au contraire sont protégés de cette fumée pat une feuille particulière (feuille ombrelle), mais ils y restent. Le garde-manger, de par sa vertu protectrice, est comme un îlot de tranquillité pour les êtres du cosmos, au sein de la maison et du village.

Ainsi l'analyse de la chaîne opératoire de fabrication du garde-manger permet-elle d'établir ses liens avec les esprits, les ancêtres et la force mana, relations qui lui confèrent une place particulière dans la maison. La technique de fabrication qui a toujours été le privilège de quelques hommes est aujourd'hui en voie de disparition. Konsis Alféa est l'un des derniers à avoir pu m'en expliciter la construction et il m'a 
aidée à déchiffrer "entre les cordelettes ", les liens sociaux complexes qui se nouent dans un objet fort banal de prime abord.

\section{BIBLIOGRAPHIE}

Balfet, Hélène

1986, « Des chaînes opératoires, pour quoi faire? », pp. 11-19, in Observer l'action technique. Paris : Éditions du CNRS.

Cabalion, Pierre

1984, «Les noms des plantes en bislamar. Origines, formations et déterminations botaniques », Journal de la Société des Océanistes 78, XL : 107-120.

Codrington, R. H.

1969, The Melanesians, Studies in their anthropology and folk-lore. Oxford : Clarendon Press (1 ${ }^{\text {ère }}$ édition 1891).

Cresswell, Robert

1976, « Techniques et culture, les bases d'un programme de travail », Techniques \& Culture $n^{\circ} 1$ (ancienne série) : 7-59.

Delaporte, Yves

1986, « Le concept de variantes dans l'analyse des chaînes opératoires ", pp. 27-30, in Observer l'action technique. Paris : Éditions du CNRS.

François, Alexandre

À paraître Dictionnaire de Mwotlap.

Mauss, Marcel

1967, Manuel d'ethnographie. Paris : Payot (« Petite bibliothèque Payot ») (1 ère édition 1947).

Rivers, W.H.

1914, The History of Melanesien society. Cambridge University Press (2 tomes).

Speiser, Felix

1996, Ethnology of Vanuatu, an early twentieth century study. Bathurst (Australia) : Crawford House Publishing (1 ${ }^{\text {ère }}$ édition 1923).

Vienne, Bernard

1982, « Les masques tamate des îles Banks », ORSTOM, Nouméa.

1984, Gens de Motlav, Idéologie et pratique sociale en Mélanésie. Paris : Musée de l'Homme (Société des Océanistes $\left.\mathrm{n}^{\circ} 42\right)$.

\section{NOTES}

2. Codrington (1891), Rivers (1914), Speiser (1921) et Vienne (1984) ont relevé diverses informations sur l'île de Mota Lava. 
3. Cette recherche de terrain visait à compléter les données de la littérature concernant le système social ancien et à comprendre celui d'aujourd'hui, après la colonisation dès le XIX $\mathrm{X}^{\mathrm{e}}$ siècle -et l'indépendance du Vanuatu en 1980, deux événements qui ont bouleversé le système traditionnel.

4. Un tronc correspond à une longueur allant d'une extrémité à l'autre des deux bras écartés d'un homme. La monnaie de coquillage a aujourd'hui totalement disparu et son déclin date de plus de 50 ans. En 1997-98, 20 Vatus équivalaient à 1 Franc (0,15 Euro) et pour prendre la mesure du niveau de vie local, un kilo de riz valait 100 Vatus (3 Euros). Un garde-manger était alors fabriqué pour très peu de monnaie, ce qui souligne le caractère non commercial de cet échange.

5. La taille réelle d'un garde-manger pouvait atteindre $2 \mathrm{~m}$ de long sur $1 \mathrm{~m}$ de profondeur.

6. Ce terme na-tmat désigne non seulement ces masques, mais aussi les défunts, les ancêtres et la constellation des Pléiades. Cette polysémie révèle le lien évident entre les masques et les ancêtres, les premiers étant l'incarnation des seconds.

7. Je reviendrai plus loin sur cette institution étudiée par ailleurs (Lanouguère-Bruneau 2002 : 306-402).

8. Une miniature du garde-manger a de nouveau été reproduite au Festival des Arts de Mota Lava, qui s'est tenu dans l'île en 2001. Sa présence témoigne de l'importance sociale et coutumière de ce meuble en voie de disparition.

9. Selon une identification faite par Alexandre François (communication personnelle : Dictionnaire de Mwotlap, en cours de rédaction).

10. La fabrication de cordes en rotin n'a pas pu être observée.

11. Identification probable selon les données relevées par Vienne (1982:586) et reprises par François (à <ATTtextecoupe>

12. Un cinquantaine d'arbres à pain ont été distingués à Mota Lava, mais aucune identification scientifique n'a encore été effectuée.

13. Le nom de ce bois n'a pas été relevé ; il est probable qu'il s'agit d'un bois tendre.

14. Il s'agit là de la seule technique relevée à Mota Lava pour protéger le bois des insectes. Il existe des plantes insecticides dans les jardins, mais elles ne sont pas utilisées dans l'artisanat et la confection d'objets à partir de végétaux

15. Le verbe ninwoy signifie "couper dans le sens de la longueur » et se différencie de totlat " couper dans le sens de la largeur».

16. La description de la matière première et de la fabrication de cette porte manque ici, faute d'observations possibles lors de mes premières enquêtes ; mais cela sera fait ultérieurement.

17. Notons également que le nom de l'arbre na-'mah désigne aussi un poisson, ce qui rappelle là encore le lien avec le monde maritime.

\section{RÉSUMÉS}

Le garde-manger est un meuble parfois encore présent dans les maisons d'habitation des îles Banks (Vanuatu), qui revêtait dans les temps anciens, une signification bien particulière. Abri dans l'abri (la maison), il permettait de stocker des biens valorisés. Il n'est plus fabriqué aujourd'hui et le savoir-faire le concernant est en voie de disparition, La description de sa fabrication technique et des matériaux utilisés permettent d'en comprendre la valeur sociale. 
One can still find a meat safe in the houses of Banks Islands (Vanuatu). It had a very special meaning in past times. A shelter inside a shelter (the house), it allowed the stocking of valuable goods. The meat safe of Mota Lava is no longer made and the knowledge of how to construct it has been given up and is going to be lost. The description of its technical making and required materials helps to understand its social value.

La fresquera es un mueble que aún existe en algunas casas de las Islas Banks (Vanuatu). Antiguamente, tenía una significación especial. Amparo dentro del amparo (la casa), permitía almacenar bienes valorizados. Hoy en día ya no se fabrica, y el saber-hacer al respecto se va desaparenciendo. La descripción de su fabricación y de las materias que utilizan permite entender su valor social.

INDEX

Mots-clés : îles Banks, maison, garde-manger, biens valorisés, artisanat, sociétés secrètes

\section{AUTEUR}

\section{VIRGINIE LANOUGUÈRE-BRUNEAU}

Doctorante EHESS, Paris 